\title{
MEANS OF ROBOTIC SUPPORT FOR ON-ORBIT SERVICING
}

\author{
Alexander Lopota, Igor Dalyaev , Igor Shardyko, Elena Kuznetcova, Ilia Belezyakov
}

Russian state scientific center for robotics and technical cybernetics (RTC), Tikhorecky prospect 21, Saint-Petersburg, 194064, Russia

\begin{abstract}
This paper presents some of the results of study into robotic on-orbit servicing (OOS) by Russian State Scientific Center for Robotics and Technical Cybernetics (RTC). Problems of OOS are presented and their importance is shown. OOS operations are briefly observed, ranged and divided into two groups. To provide these operations two design concepts for means of robotic support (MRS), placed on servicing satellite, are considered. Those are design concepts of MRS based on one manipulator system (MRS-1) and MRS with three manipulators (MRS-3), more detailed description of them is also given.
\end{abstract}

Keyword: satellite; on-orbit servicing; active lifetime; space vehicle; robotic support
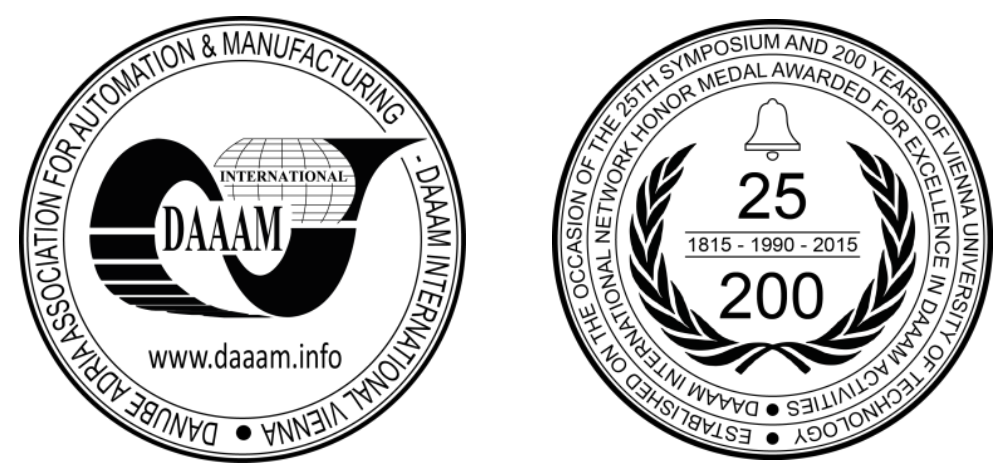

This Publication has to be referred as: Lopota, A[lexander]; Dalyaev, I[gor]; Shardyko, I[gor]; Kuznetcova, E[lena] \& Belezyakov, I[lia] (2016). Means of Robotic Support for On-Orbit Servicing, Proceedings of the 26th DAAAM International Symposium, pp.0865-0870, B. Katalinic (Ed.), Published by DAAAM International, ISBN 978-3-90273407-5, ISSN 1726-9679, Vienna, Austria

DOI:10.2507/26th.daaam.proceedings. 120 


\section{Introduction}

Development, manufacturing and launching of space vehicles are very expensive, so there is currently an urgent need in extension of their active lifetime. International experience analysis shows that the most valuable and unique objects, such as "Hubble" space telescope, have been repeatedly maintained and repaired in outer space by astronauts. It should be noted that such expeditions entail a certain risk to the life of manned space vehicle crews and have an extremely high cost. It is obvious that such operations should be preferably carried out by servicing satellite equipped with MRS [1].

World powers performed developments aimed at creating service spacecrafts, which will carry out orbital maintenance operations on functioning space vehicles. When creating service satellite much attention is payed to such tasks as inspection [2, 3, 4, 5], transportation [6], motion control, refueling, maintenance and repair work, carrying out orbital assembly.

In 2007 the space experiment was conducted under Orbital Express program [7, 8], which aim to develop autonomous technology of refueling and reconfiguration of satellites directly in orbit. The experiment simulated a rendezvous of two launched satellites - ASTRO to NextSat - in automatic mode with the flyby maneuvers in close proximity (approximately $60 \mathrm{~m}$ ), a soft catching, docking, fuel transfer, replacement of batteries and computers, bringing them into operation, undocking of space vehicles.

Now under the auspices of DARPA there is continuing work within FREND program [9, 10] pursuing the creation of multi-service satellites, equipped with a robotic system of three manipulators, allowing ducking with an optional satellite with no docking interfaces. Besides on Phoenix space platform development [11, 12], which will grab dead satellites, dismantle their antennas and other equipment, is underway. To each of the dismantled part or unit a tiny spacecraft will be attached; original engine and ID will keep the unit at a given point in space. Thus, it will form a warehouse of parts for space satellites, which can be retrieved when needed.

Currently SpaceTech GmbH continues working on the creation of multi-service spacecraft DEOS [13, 14, 15]. The main objectives are to provide rendezvous of service satellite with serviceable spacecraft, docking and relative orientation of satellites using service satellite's manipulator, docking with the spacecraft for replacement of equipment units or refueling, ensure a controlled descent from orbit or launch into the desired orbit and spacecraft flooding at the end of the mission.

Company Tethers Unlimited [16] continues to research and develop SpiderFab technology, allowing space platforms to use three-dimensional printing and robotic assembly for creating and combining large structures in orbit such as space antenna, solar panels and shields which are ten times as larger as existing folding options. By using this technology, the volume of cargo to be delivered into orbit will decrease in 30 times with the same weight, and total system size will be doubled.

Based on the stated above, we can claim that the search for technical solutions for the creation of servicing satellites equipped with MRS [17], which are able to perform an orbital spacecraft service operations, is a relevant for on-orbit servicing and high demand task.

\section{Analysis, classification and systematization of orbital servicing operations}

Timely maintenance of space vehicles provides a qualitative implementation of their tasks for a longer time. The probability of onboard equipment failure may be compensated by the possibility of its maintenance and repair during in orbit spacecraft exploitation.

Servicing spacecraft tasks can range from the relatively simple task of inspection (remote and contact) to complex maintenance tasks such as refueling of uncooperative space vehicle. The entire set of orbital service operations can be divided into three main categories [18]: the elimination of failure, lifetime extension and other operations. The operations of failure elimination include:

- motion control: orbit correction - moving client spacecraft from incorrect initial delivery orbit;

- maintenance and repair work: deployment - assistance in deploying of solar panels, antennas and other deployable items;

- maintenance and repair work: repair or replacement of failed components.

- Extending the spacecraft lifetime is possible with the following service operations:

- motion control: transportation - transfer of client spacecraft to a new work orbit to save fuel reserves;

- replenishment of consumables, refueling - replenishment of fuel, coolant and other consumable materials;

- maintenance and repair work: component replacement - replacement of worked-out elements, - also modernization in order to expand the functionality of satellite and reconfiguration.

- In addition to elimination of failures and lifetime extension operations following service operations are possible:

- inspection (remote or contact) - inspection of client spacecraft with purpose of troubleshooting;

- motion control: de-orbiting (disposal/flooding) - moving of deenergized client spacecraft and space debris from working orbit to disposal orbit or into the atmosphere;

- $\quad$ assembly operations - assembly of truss structures, space vehicles and space stations;

- disassembly - removal of functional units of worked-out spacecrafts in order to replace defective elements of malfunctioning space vehicles. 
The operations mentioned above, except for remote inspection, imply docking of service satellite to client spacecraft. Docking with cooperative spacecraft can be realized without means of robotic support, since since special docking devices to perform the operation using satellite's propulsion system are allowed for in the design of service satellite. The docking of service satellite with uncooperative space vehicle can be carried out either by means of constructive elements of the latter, for example, the apogee motor nozzle, or using means of robotic support. Technical configuration definition for service spacecraft's MRS, handling with uncooperative space units, should be carried out in each case individually due to the lack of standardized, comfortable to grip and solid client spacecraft's constructive elements.

The presence or absence of manipulators, embodied in means of robotic support, and their number depends on the operations carried out by service satellite and type of serviced vehicle. It should be noted that all operating satellites in orbit are currently uncooperative. At the same time, in contrast to foreign space vehicles, the majority of Russian satellites have no apogee motor nozzles since they are inserted into high orbits with special upper stage of "Breeze-M". Therefore, presence of manipulator system on service satellite for maintenance of Russian on-orbit) spacecrafts is required.

Generally, in terms of need for means of robotic support of service satellite, all the operations can be divided into two groups:

- operations that require means of robotic support in the form of manipulator system:

a) contact inspection;

b) transportation (disposal) of uncooperative spacecrafts using means of robotic support;

c) reconfiguration, modernization, replacement of functional units;

d) maintenance and repair work;

e) replenishment of consumables;

f) assembly operations using means of robotic support;

g) dismantling of spacecraft into its component parts.

- operations that can be performed without means of robotic support of service satellite:

a) remote inspection;

b) transportation by means of special docking station (in relation to cooperative space vehicles);

c) refueling of spacecrafts [19] with dedicated docking device interface for fuel transfer (in relation to cooperative spacecrafts);

d) assembly of large space structures consisting of major target modules using their propulsion units (in relation to cooperative spececrafts).

Thus, operations of the second group can be performed only with cooperative space vehicles, which are not under development yet. An exception is the remote inspection that can be carried out by any service satellites.

\section{The composition and structure of means of robotic support of service spacecraft}

A space robotic system (also referred to as space manipulator or space robot) for an OOS mission typically consists of three major components: the base spacecraft or servicing satellite, an n-degree-of-freedom (n-DoF) robot manipulator attached to the servicing satellite, and the target spacecraft to be serviced [20].

Execution of operations described in previous section is possible by various service satellites, thus composition options will be different in operation quality performance, applied technologies level, amount of material and time resources required for the development and production of proper means of robotic support. Generally, means of robotic support should be composed of a base unit, manipulator system, observation unit, changeable tools dispenser, payload cell, docking device. To maintain the operating temperature range in outer space, means of robotic support should have a common thermal control system. Power supply and communication with ground or orbital control center are realized by means of service spacecraft.

In RTC there were developed two design concepts of MRS based on one manipulator system (MRS-1) and MRS with three manipulators (MRS-3), descriptions of which are given below. Approximate weight of MRS-1 is 318 $\mathrm{kg}$, MRS-3 - $548 \mathrm{~kg}$. The presence of three manipulators composing MRS-3 makes it possible to maintain both cooperative and uncooperative space vehicles. Service satellite equipped with MRS-1 may serve spacecrafts, docking to which be realized with the docking device of MRS-1. General view of MRS-1 is shown in Figure 1. 


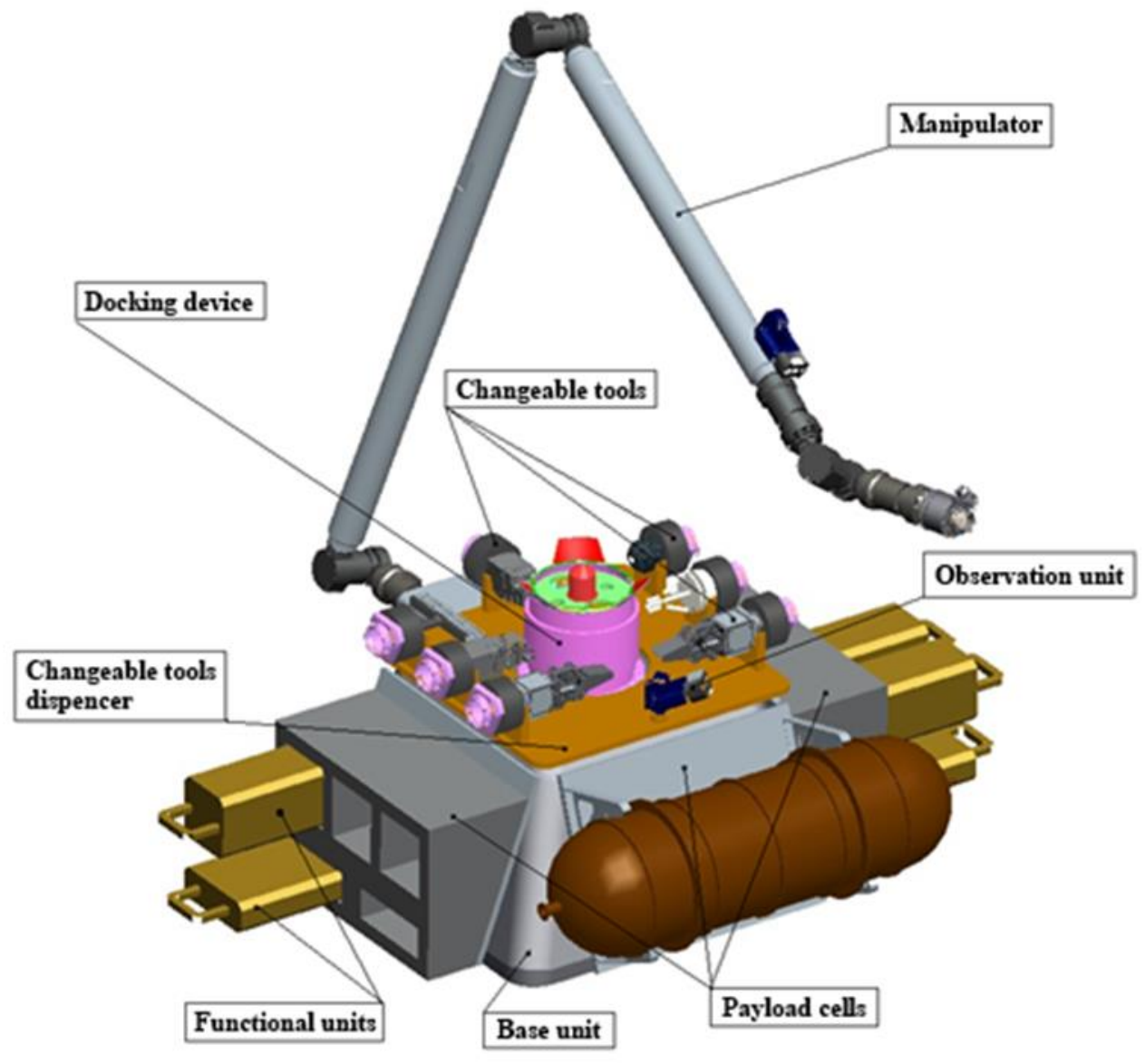

Fig. 1. General view of MRS-1 with use of

The main element of MRS-1 is a 6-DoF manipulator sufficient to perform necessary operations. Optionally, 7-DoF manipulator arm the service area will expand and avoidance of various obstacles will be possible. Manipulator ends with tool fixation device.

Changeable tool dispenser is located on top of base unit. The dispenser has necessary removable tools for manipulator; the tools are fixed in the dispenser fastening devices. The manipulator is attached to one side of base block, from other three sides of which the payload cells with fixed functional units and an expansion tank (for refueling operations) are anchored. Docking device is located in the middle of and higher than dispenser, so that after docking with client spacecraft manipulator could freely change tools.

Performing of target operations begins with the grip of necessary tool from the dispenser. After seizing the tool by manipulator and connecting of suitable electrical connectors fastening device unlocks and changeable tool can be freely extracted for operation execution.

Contact inspection involves work of manipulator to identify various problems. As an option - cutting thermal blanket or another kind of mechanical impact on client space vehicle for visual access to the interior equipment.

On both sides of the base unit payload cells are arranged to accommodate functional blocks. One of them is to store breakdown or obsolete function blocks taken from client spacecrafts during the repair work, the other - for new units.

It is essential that replacement of functional units operations are only possible with promising cooperative spacecrafts specifically designed to allow this operation. Performing of these operations with currently orbiting space vehicles is impossible.

The fuel tank is located on the base block opposite to the manipulator. It is intended to refuel serviced spacecrafts. The refueling operation is carried out without manipulator with the special connection device interface. Thus, this operation is only possible with cooperative space vehicles.

Orbital assembly operations with use of one manipulator can be implemented in two scenarios: the assembly takes place directly on the target client spacecraft (building extra sections, deployment of solar panels or antennas); or assembly takes place in segments on board of the service spacecraft with subsequent installation of finished segments on client space vehicle.

General view of MRS-3 is shown in Figure 2. MRS-3 differs from MRS-1 by presence of two additional identical 6 DoF manipulators. 


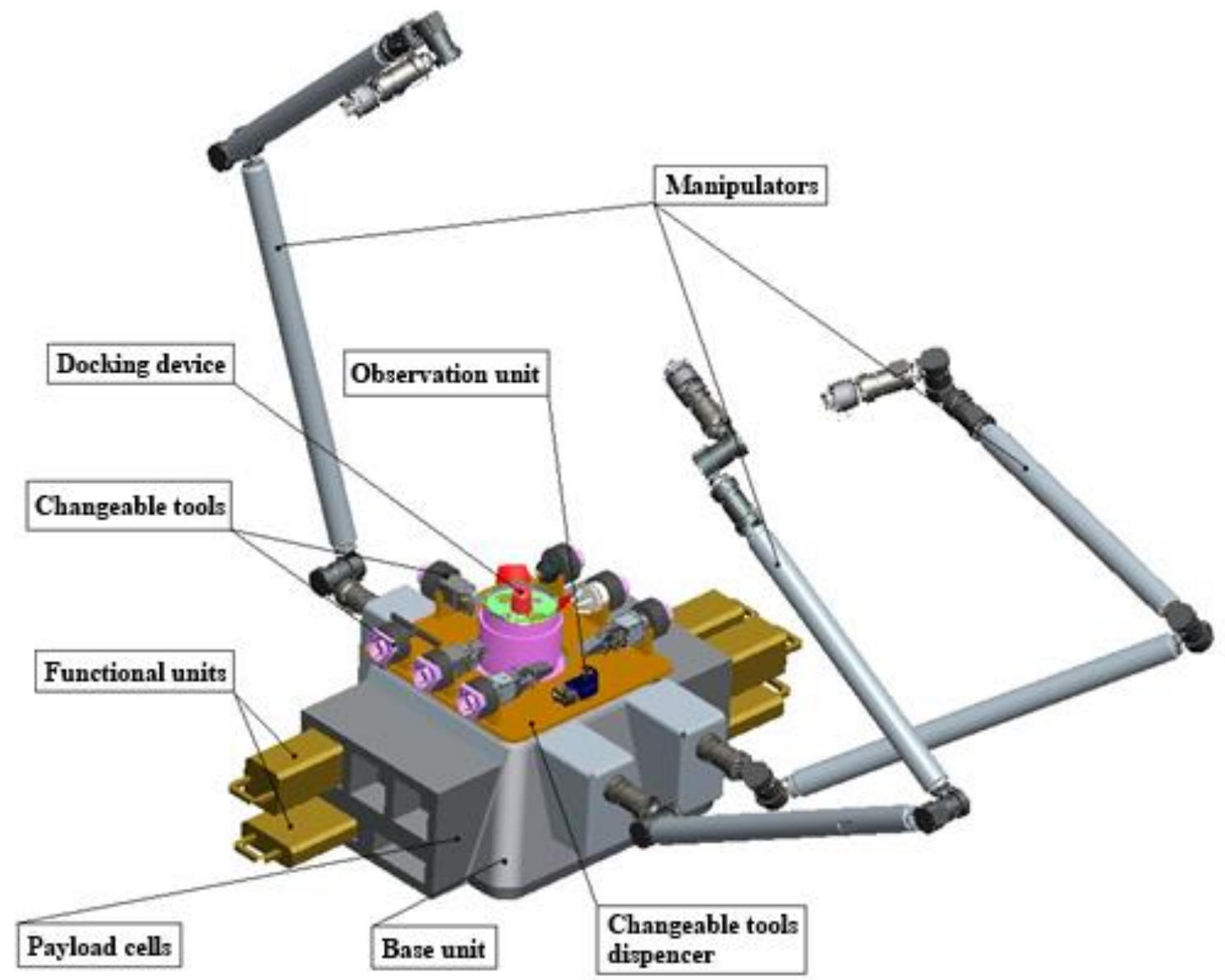

Fig. 2. General view of MRS-3

All manipulators, as well as in MRS-1, end with tool fixation device. Additional manipulators are located instead of the fuel tank, so service spacecraft performs refueling operation by pumping its own fuel.

Performing of target operations, as in case of MRS-1, begins with the capture of necessary tool from changeable tools dispenser. Contact inspection operation is conducted by manipulators teamwork and can be done much faster than in case of MRS-1, because it doesn't require a tool change on each step.

Operation of functional units replacement with MRS-3 is no different from its execution by MRS-1, only MRS-3 can perform it faster.

The presence of three technological manipulators in MRS may require more changeable tools within the same nomenclature. Manipulators in stowed position are folded along the body of service spacecraft not overlapping overall dimensions.

\section{Conclusion}

Analyzing design features of developed robotic systems, it can be concluded that MRS-1 is able to perform all necessary orbital service operations with either cooperative objects, or uncooperative objects or uncooperative objects, which construction include the elements like the apogee motor nozzle, which make possible to use the docking device for (to provide the) space link. Service satellite, equipped with MRS-1, when launching is able to carry a larger number of functional blocks and fuel supply than MRS-3.

MRS-3 is able to maintain cooperative and uncooperative space vehicles of optional design. Some orbital maintenance operations of cooperative spacecrafts, for example, replacement of functional units service satellite equipped with MRS-3 can perform faster. However, such satellite performs refueling operation by its own fuel supply, which reduces its lifetime. The fuel tank fixed on payload cell of MRS-1 allows to refuel more client spacecrafts.

Thus, maintenance of currently operated domestic satellites can only be performed by service satellites with MRS-3 after improvement of their identified drawbacks.

RTC is prepared to manufacture prototypes of MRS for OOS missions within the next few years.

The applied scientific investigations are carried out with the financial support of the Russian Federation represented by the Russian Ministry of Education and Science. The unique identifier of the applied scientific investigations is RFMEFI57814X0046. 


\section{References}

[1] Ellery A, Kreisel J, Sommer B. The case for robotic on-orbit servicing of spacecraft: spacecraft reliability is a myth. Acta Astronaut 2008.

[2] MiTEx A [Electronic source] / Free access: http://space.skyrocket.de/doc_sdat/mitex-a.htm, free. - Screen title.

[3] MiTEx B [Electronic source] / Free access: http://space.skyrocket.de/doc_sdat/mitex-b.htm, free. - Screen title.

[4] XSS (Experimental Spacecraft System), XSS-10 \& XSS-11 Missions [Electronic source] / Free access: https://directory.eoportal.org/web/eoportal/satellite-missions/v-w-X-y-z/xss, free. - Screen title.

[5] Oda M, Kibe K, Yamagata F. ETS-VII, space robot in-orbit experiment satellite. In: IEEE international conference on robotics and automation. vol. 1, Minneapolis, MN; 1996.

[6] CX-OLEV [Electronic source] / Free access: https://artes.esa.int/projects/conexpress-orbital-life-extensionvehicle-cX-olev, free. - Screen title.

[7] Orbital Express Space Operations Architecture [Electronic source] / Free access: http://archive.darpa.mil/orbitalexpress/index.html, free. - Screen title.

[8] Mulder T. Orbital express autonomous rendezvous and capture flight operations. In: AIAA/AAS astrodynamics specialist and exhibit, Honolulu, Hawaii; 2008.

[9] Kelm, B.E. FREND: Pushing the Envelope of Space Robotics [Electronic source]/ B.E. Kelm. - Space research and satellite technology, 2008 -. - Access mode: http://www.nrl.navy.mil/content_images/08Space_Kelm.pdf, free. - Screen title.

[10] Kelm B, Angielski J, Butcher S, Creamer N, Harris K, Henshaw C, et al. FREND: pushing the envelope of space robotics. NRL Rev-Space Res 2008.

[11] Phoenix [Electronic source] / Free access: http://www.darpa.mil/our_work/tto/programs/phoenix.aspx, free. Screen title.

[12] TSullivan B, Hunter R, Bruhn J, Fowler E, Hoag L, Chappie S, et al. Phoenix project status 2013. In: AIAA SPACE conference \& exposition, San Diego, CA; 2013. p. 1-17.

[13] DEOS [Electronic source] / Free access: http://www.spacetech-i.com/deutsche-orbitale-servicing-mission.html, free. - Screen title.

[14] Reintsema D, Thaeter J, Rathke A, Naumann W, Rank P, Sommer J. DEOS the German robotic approach to secure and de-orbit malfunctioned satellites from low earth orbit. In: International symposium on artifical intelligence, robotics and automation in space, Sapporo, Japan; 2010. p. 244-251.

[15] Reintsema D, Sommer J, Wolf T, Thaeter K, Radthke A, Naummann W, et. al. DEOS-the in-flight technology demonstration of German robotics approach to dispose malfunctioned satellites. In: ESA workshop on advanced space technologies for robotic and automation, ESTEC, Noordwijk, The Netherlands; 2011.

[16] Tethers Unlimited [Electronic source] / Free access: http://www.tethers.com/SpiderFab.html, free. - Screen title.

[17] Sabatini M, Monti R, Gasbarri P, Palmerini G. Deployable space manipulator commanded by means of visualbased guidance and navigation, Acta Astronaut 2013; 83:27-43.

[18] Sullivan Rowland B. Technical and economic feasibility of telerobotic on-orbit satellite servicing - University of Maryland Space Systems Laboratory, 2005.

[19] Lavagna M, Bucci L, Cavenago F, Rivolta A. Robotic refueling system for space platform servicing. In conference: 66th international astronautical congress, at Jerusalem. October 2015.

[20] Angel Flores-Abad, Ou Ma, Khanh Pham, Steve Ulrich. A review of space robotics technologies for on-orbit servicing. In: Progress in Aerospace Sciences, Volume 68, July 2014, p. 1-26. 